\section{Adaptação transcultural do instrumento Appraisal of Guidelines for Research \& Evaluation II (AGREE II) para avaliação de diretrizes clínicas}

\author{
Cross-cultural adaptation of the instrument \\ Appraisal of Guidelines For Research \& Evaluation \\ II (AGREE II) for assessment of clinical guidelines
}

\section{Adaptación transcultural del instrumento \\ Appraisal of Guidelines for Research \& Evaluation II (AGREE II) para la evaluación de directrices clínicas}

\begin{abstract}
This article describes the adaptation of the Appraisal of Guidelines for Research \& Evaluation II (AGREE II) instrument into Portuguese. A cross-cultural adaptation followed internationally accepted standards with the stages of translation, synthesis, back-translation, evaluation by an expert committee, and external evaluation. The translated instrument was published on the official AGREE websites.
\end{abstract}

Practice Guideline; Evaluation of Research

Programs and Tools; Translating
Gleide Simas Custódio Khan 1,2

Airton Tetelbom Stein 2,3

\section{Resumo}

$O$ artigo tem por objetivo descrever o processo de adaptação transcultural do instrumento Appraisal of Guidelines for Research \& Evaluation II (AGREE II) para o português do Brasil. A adaptação seguiu as normas internacionais com as etapas de tradução, síntese, retrotradução, avaliação por um comitê de especialistas e avaliação externa. O instrumento traduzido foi publicado no site do órgão oficial de gestão dos interesses do instrumento.

Guia de Prática Clínica; Avaliação de Programas e Instrumentos de Pesquisa; Tradução 


\section{Introdução}

Inúmeras organizações em diversos países do mundo têm se dedicado à sistematização de diretrizes clínicas com o objetivo de melhorar a qualidade da assistência prestada ao paciente.

Diretriz clínica é um conjunto de afirmações desenvolvidas de forma sistematizada para apoiar as decisões do clínico e do paciente acerca dos cuidados de saúde mais apropriados em circunstâncias clínicas específicas 1 .

A qualidade de uma diretriz clínica está diretamente relacionada à confiança de que os vieses potenciais do desenvolvimento foram adequadamente abordados, que as orientações são exequíveis, que apresente validades interna e externa, além de levar em consideração os benefícios, riscos e custos das recomendações ${ }^{3}$. Deve estar apoiada em revisões sistemáticas da literatura e isentas de vieses induzidos por interesses comerciais ou corporativos.

Existem diretrizes clínicas que não contemplam boa parte dos requisitos necessários. São formuladas fora dos ambientes das grandes agências de avaliação de tecnologias em saúde (ATS) e instituições governamentais, carecendo de avaliações mais rigorosas de instrumentos de avaliação consensuados e empregados pela comunidade internacional 4,5.

O instrumento Appraisal of Guidelines for Research \& Evaluation (AGREE) foi elaborado para abordar a variabilidade na qualidade das diretrizes. É uma ferramenta que avalia o rigor metodológico e a transparência com que uma diretriz clínica é desenvolvida 3,6. A ferramenta possibilita o julgamento sobre o método utilizado para elaborar as diretrizes clínicas, o conteúdo das recomendações finais e os fatores que estão ligados à sua aceitação.

\section{Método}

O AGREE II consiste em uma ferramenta de 23 itens, abrangendo seis domínios de qualidade com uma escala Likert de 1 a 7 (1: discordo totalmente a 7: concordo totalmente) para pontuação de cada item. Os seis domínios são: escopo e finalidade, envolvimento das partes interessadas, rigor do desenvolvimento, clareza e apresentação, aplicabilidade e, por último, independência editorial (Tabela 1).

O domínio "escopo e finalidade" diz respeito ao objetivo geral da diretriz, às questões específicas de saúde e à população-alvo. O "envolvimento das partes interessadas" avalia o grau em que a diretriz representa os pontos de vista de seus usuários e se os indivíduos de todos os grupos profissionais relevantes foram representados. O domínio "rigor do desenvolvimento" avalia especificamente se os métodos sistemáticos foram usados para a busca de evidências. A "clareza da apresentação" diz respeito à linguagem, estrutura e ao formato da diretriz. Já o domínio "aplicabilidade" avalia questões que são pertinentes à implementação da diretriz, considera as barreiras organizacionais e os recursos relacionados à sua utilização. A "independência editorial” considera os conflitos de iteresse na formulação das recomendações.

$\mathrm{O}$ instrumento foi traduzido seguindo as normas metodológicas recomendadas 7,8. As etapas foram: tradução, síntese, retrotradução, revisão pelo grupo de trabalho e revisão externa por especialistas.

A tradução do instrumento do inglês para o português foi feita por dois tradutores independentes. Com base na análise das traduções independentes foi obtida uma versão comum das traduções (síntese), sendo então encaminhada para a retrotradução.

Nas duas retrotraduções independentes, os dois retrotradutores não tiveram nenhum conhecimento sobre os conceitos envolvidos no instrumento e não participaram da primeira tradução.

As duas retrotraduções foram comparadas com a versão original do AGREE II, estabelecendo-se a versão definitiva da tradução. Essa versão foi encaminhada para nove experts em diretrizes clínicas ligados a agências de ATS, universidades e um consultor do Ministério da Saúde, com o intuito de identificar inconsistências e melhorar o formato de comunicação.

Acatadas as alterações sugeridas, foi encaminhada à avaliação pelo AGREE Research Trust, sujeita à aprovação.

O grupo de coordenação da tradução foi composto por uma enfermeira (G.S.C.K.) e dois médicos (epidemiologista e internista). Todas as etapas do processo de tradução foram revisadas pelo grupo coordenador.

\section{Resultados e discussão}

Há consenso de que a implementação de diretrizes clínicas a partir das melhores evidências científicas disponíveis produz melhores resultados na população assistida. Melhoram a assistência prestada com a padronização das condutas frente a problemas clínicos idênticos, independentes de onde ou por quem sejam tratados. Além de subsidiarem as decisões dos profissionais de saúde, as diretrizes desempenham um papel importante para a gestão e regulação dos sistemas de saúde 9,10 . 
Tabela 1

Itens do Appraisal of Guidelines for Research \& Evaluation II (AGREE II).

\begin{tabular}{|c|c|c|}
\hline \multirow{2}{*}{$\begin{array}{l}\text { Domínios } \\
\text { 1. Escopo e finalidade }\end{array}$} & \multicolumn{2}{|l|}{ Itens } \\
\hline & 1. & O(s) objetivo(s) geral(is) da(s) diretriz(es) está(ão) especificamente descrito(s). \\
\hline & 2. & A(s) questão(ões) de saúde coberta(s) pela diretriz está(ão) especificamente descrita(s). \\
\hline & 3. & A população (pacientes, público etc.) a quem a diretriz se destina está especificamente descrita. \\
\hline \multirow[t]{3}{*}{ 2. Envolvimento das partes interessadas } & 4. & A equipe de desenvolvimento da diretriz inclui indivíduos de todos os grupos profissionais relevantes. \\
\hline & 5. & Procurou-se conhecer as opiniões e preferências da população-alvo (pacientes, público etc.). \\
\hline & 6. & Os usuários-alvo da diretriz estão claramente definidos. \\
\hline \multirow[t]{8}{*}{ 3. Rigor do desenvolvimento } & 7. & Foram utilizados métodos sistemáticos para a busca de evidências. \\
\hline & 8. & Os critérios para a seleção de evidências estão claramente descritos. \\
\hline & 9. & Os pontos fortes e limitações do corpo de evidências estão claramente descritos. \\
\hline & 10. & Os métodos para a formulação das recomendações estão claramente descritos. \\
\hline & 11. & $\begin{array}{l}\text { Os benefícios, efeitos colaterais e riscos à saúde foram considerados na formulação das } \\
\text { recomendações. }\end{array}$ \\
\hline & 12. & Existe uma relação explícita entre as recomendações e as evidências que lhe dão suporte. \\
\hline & 13. & A diretriz foi revisada externamente por experts antes da sua publicação. \\
\hline & 14. & Um procedimento para atualização da diretriz está disponível. \\
\hline \multirow[t]{3}{*}{ 4. Clareza da apresentação } & 15. & As recomendações são específicas e sem ambiguidade. \\
\hline & 16. & $\begin{array}{l}\text { As diferentes opções de abordagem da condição ou problema de saúde estão claramente } \\
\text { apresentadas. }\end{array}$ \\
\hline & 17. & As recomendações-chave são facilmente identificadas. \\
\hline \multirow[t]{4}{*}{ 5. Aplicabilidade } & 18. & A diretriz descreve os fatores facilitadores e as barreiras para a sua aplicação. \\
\hline & 19. & $\begin{array}{l}\text { A diretriz traz aconselhamento e/ou ferramentas sobre como as recomendações podem ser colocadas } \\
\text { em prática. }\end{array}$ \\
\hline & 20. & $\begin{array}{l}\text { Foram consideradas as potenciais implicações quanto aos recursos decorrentes da aplicação das } \\
\text { recomendações. }\end{array}$ \\
\hline & 21. & A diretriz apresenta critérios para o seu monitoramento e/ou auditoria. \\
\hline \multirow[t]{2}{*}{ 6. Independência editorial } & 22. & O parecer do órgão financiador não exerceu influência sobre o conteúdo da diretriz. \\
\hline & 23. & $\begin{array}{l}\text { Foram registrados e abordados os conflitos de interesse dos membros da equipe que desenvolveram } \\
\text { a diretriz. }\end{array}$ \\
\hline
\end{tabular}

Essa ferramenta foi delineada para avaliar as diretrizes desenvolvidas por grupos locais, regionais, nacionais ou internacionais para qualquer estágio do cuidado em saúde, incluindo a promoção, saúde pública, rastreamento, diagnóstico ou tratamento 3 .

Destina-se aos profissionais de saúde que desejam avaliar uma diretriz antes de adotar as recomendações em sua prática e aos elaboradores de diretrizes para seguirem uma metodologia de desenvolvimento estruturada e rigorosa.

Outro instrumento relacionado à avaliação de diretrizes é o Grading of Recommendations Assessment, Development, and Evaluation (GRADE). Esse apresenta uma abordagem que classifica a direção e a força das recomendações. É um instrumento fundamental para os elaboradores, no qual os métodos e processos para avaliar as evidências são essenciais para a qualidade das diretrizes clínicas 11.
Uma fragilidade que é possível identificar no instrumento AGREE II é a sua complexidade, uma vez que os profissionais necessitam de tempo e domínio da ferramenta para a sua aplicação. Em contrapartida, apresenta vantagens de ser uma lista de checagem que facilita o processo de planejamento, elaboração e implementação de diretrizes, podendo ser utilizado por profissionais de diferentes áreas da saúde.

Esse instrumento foi traduzido para o português do Brasil, seguindo todas as etapas recomendadas pelo AGREE Research Trust. Na etapa da retrotradução foram corrigidas algumas frases relacionadas à semântica e gramática, a fim de tornar o texto adequado à língua portuguesa. Foi publicado no site oficial do órgão de gestão dos interesses do instrumento (http://www.agree trust.org).

Nesse contexto, a disponibilidade do AGREE II, adaptado para a língua portuguesa do Brasil, 
poderá auxiliar os profissionais da saúde envolvidos tanto na elaboração quanto na utilização de diretrizes clínicas, contribuindo, assim, no processo de qualificação do cuidado de saúde.

\section{Resumen}

El artículo tiene como objetivo describir el proceso de adaptación transcultural de la herramienta de trabajo Appraisal of Guidelines for Research \& Evaluation II (AGREE II) al portugués de Brasil. La adaptación siguió las normas internacionales para las etapas de traducción, sintesis, retrotraducción, evaluación por un comité de expertos y evaluación externa. El instrumento traducido resultante se publicó en el sitio web del órgano oficial de gestión, relacionado con los objetivos del mismo.

Guía de Práctica Clínica; Evaluación de Programas e Instrumentos de Investigación; Traducción

\section{Colaboradores}

G. S. C. Khan e A. T. Stein participaram da concepção, redação do artigo e revisão da versão a ser publicada.

\section{Agradecimentos}

Ao Núcleo de Avaliação de Tecnologias em Saúde do Grupo Hospitalar Conceição. Ao DECIT/Ministério da Saúde, pelo financiamento.

\section{Referências}

1. Graham R, Mancher M, Wolman DM, Greenfield S, Steinberg E. Clinical practice guidelines we can trust. Washington DC: National Academies Press; 2011.

2. Agência Nacional de Saúde Suplementar. A implementação de diretrizes clínicas na atenção à saúde: experiências internacionais e o caso da saúde suplementar no Brasil. Brasília: Organização PanAmericana da Saúde/Rio de Janeiro: Agência Nacional de Saúde Suplementar; 2009.

3. Cluzeau FA, Browers M, Grol R, Makela M, Littlejohns P, Grimshaw J, et al. Development and validation of an international appraisal instrument for assessing the quality of clinical practice guidelines: the AGREE project. Qual Saf Health Care 2003; 12:18-23.

4. Ribeiro RC. Diretrizes clínicas: como avaliar a qualidade? Rev Soc Bras Clín Méd 2010; 8:350-5.

5. Qaseem A, Forland F, Macbeth F, Ollenschläger G, Phillips S, van der Wees P. Guidelines international network: toward international standards for clinical practice guidelines. Ann Intern Med 2012; 156:525-31.

6. Brouwers MC, Kho ME, Browman GP, Burgers JS, Cluzeau F, Feder G, et al. Development of the AGREE II, part 2: assessment of validity of items and tools to support application. CMAJ 2010; 182:E472-8.

7. Guillemin F, Bombardier C, Beaton D. Cross-cultural adaptation of health-related quality of life measures: literature review and proposed guidelines. J Clin Epidemiol 1993; 46:1417-32.

8. Beaton D, Bombardier C, Guillemin F, Ferraz MB Recommendations for the cross-cultural adaptation of health status measures. New York: American Academy of Orthopaedic Surgeons; 2002.

9. Grol R. Successes and failures in the implementation of evidence-based guidelines for clinical practice. Med Care 2001; 39(8 Suppl 2):II46-54.

10. Schmidt MI, Duncan BB. Epidemiologia clínica e medicina baseada em evidências. In: Rouquayrol MZ, Almeida Filho N, organizadores. Epidemiologia \& saúde. 6a Ed. Rio de Janeiro: Editora Medsi; 2003. p. 193-228.

11. Andrews J, Guyatt G, Oxman AD, Alderson P, Dahm P, Falck-Ytter Y, et al. GRADE guidelines: going from evidence to recommendations: the significance and presentation of recommendations. J Clin Epidemiol 2013; 66:719-25.

Recebido em 09/Dez/2012

Versão final reapresentada em 12/Dez/2013 Aprovado em 25/Fev/2014 